\title{
¿Qué es eso de la interseccionalidad? Aproximación al tratamiento de la diversidad desde la perspectiva de género en España
}

\author{
Carmen EXPÓSITO MOLINA \\ Grupo de Investigación Multiculturalismo y Género \\ Universidad de Barcelona \\ karmexpo@gmail.com
}

Recibido: 20.12.2011

Aceptado: 01.03.2012

\section{RESUMEN}

El desarrollo de las políticas de diversidad y de las políticas de igualdad de género en España ha tenido una evolución paralela en el tiempo. Las mujeres inmigradas han estado en buena medida ignoradas desde las políticas de diversidad y poco consideradas desde las políticas de igualdad. En los últimos años, no obstante, estas últimas han empezado a incorporarlas desde los diferentes ámbitos. En el momento actual un cambio de interpretación de las desigualdades ha hecho emerger el concepto de interseccionalidad como el instrumento para abordar adecuadamente sus realidades y situaciones de vida.

Palabras clave: inmigración, perspectiva de género, transversalidad, desigualdad, interseccionalidad.

\section{What is that of Intersectionality? \\ Approach to Dealing with Diversity from a Gender Perspective in Spain}

\begin{abstract}
The development of diversity policies in Spain has had a parallel evolution at the same time as gender equality policies. Immigrant women have been largely ignored in diversity policies and rarely considerate from equality policies. In recent years, however, the latter have started to incorporate them into different areas. At present a change of interpretation of inequalities made emerged the concept of intersectionality as a tool to approach adequately their address and their realities life situations.
\end{abstract}

Key words: immigration, gender, mainstreaming, inequality, intersectionality.

"Puede haber también algún acoplamiento de desventajas entre diferentes fuentes de privación, y esto puede ser de importancia crítica para entender la pobreza y formular políticas públicas para afrontarlas". (Amartya Sen, 2010)

\section{INTRODUCCIÓN}

En el Estado español el despliegue de las políticas para gestionar la inmigración ha sido casi paralelo al desarrollo de las políticas de igualdad de género. 
En el año 1975 España pasa de ser un estado de hecho a un estado de derecho y empieza a definirse orgánicamente. Se inician nuevos servicios y estructuras de atención a la población.

Las mujeres habían sido ya activas durante los últimos años de la dictadura, consiguiendo el reconocimiento de la discriminación por razón de sexo en la Constitución de 1978. Antes en 1976 habían tenido lugar en Barcelona las Jornadas Catalanas de la Mujer, consideradas el primer acto de discusión colectiva del feminismo. En 1983 se crea el Instituto de la Mujer y en 1988 se implementa el I Plan para la Igualdad de Oportunidades.

En 1985 se aprobaba la Ley Orgánica 7/1985 sobre derechos y libertades de los extranjeros residentes en España, conocida como la Primera Ley de Extranjería. A partir de este momento las políticas de extranjería y las políticas de igualdad de género fueron evolucionando cada una por su lado, de acuerdo con la realidad y nuevas necesidades, así como en consonancia con las directrices adoptadas en la Comunidad Europea.

Las políticas de extranjería han sido en buena medida neutras al género y se puede afirmar que las mujeres inmigradas han entrado en el discurso oficial de la mano de las políticas de igualdad de género, aunque en España debido a su contexto sociopolítico específico primó en los primeros años de la democracia la categoría universal de mujer. En la actualidad se aprecia desde las políticas públicas europeas y españolas un cambio de enfoque en relación al tratamiento de las desigualdades entre las que se incluyen el género y la raza o etnia. En este contexto desde la perspectiva de género la interseccionalidad ha ido cobrando protagonismo dentro del discurso académico como método de interpretación y abordaje de las desigualdades que afectan a las mujeres.

"L'intersectionnalité analyse comment intergisent des types spécifiques, construits historiquement, de distributions inégales du pouvoir et/ou de normativités contraignantes, fondées sur des catégorisations socio-culturelles construites discursivement, institutionnellement et/ou structurellement telles que le genre, l'ethnicité, la race, la clase, la sexualité, l'âge ou la génération, le hándicap, la nationalité, la langue maternelle, etc, de sorte à produire différents types d'inégalités sociales" (Kóczé, 2011:134). 
La transversalidad ${ }^{1}$ ha representado, posiblemente, la estrategia política más importante que ha permitido abordar la desigualdad de género desde todos los ámbitos, todos los procesos y todos los niveles e incorporar la experiencia de las mujeres en el diseño, implementación, desarrollo y evaluación de las políticas. Con la incorporación de la diversidad y el concepto de desigualdades/discriminaciones múltiples habrá que ver si el género seguirá ocupando el lugar preeminente que ha ocupado hasta ahora por un lado, y por el otro cómo se ubican las mujeres inmigradas en este nuevo escenario. ¿Serán consideradas en función de su origen?, como han venido haciendo las políticas de diversidad. ¿O la aproximación interseccional será incorporada al ámbito político y permitirá dar respuestas a las necesidades de las mujeres en función del género?

\section{DEL RECONOCIMIENTO DE LA DIVERSIDAD A LA IGUALDAD DE TRATO Y LA NO DISCRIMINACIÓN. DE LA TRANSVERSALIDAD A LA INTERSECCIONALIDAD}

España ha sido pionera en políticas de Igualdad (Forest, Platero, 2008) y por lo tanto la igualdad de género ha tenido un enfoque unitario ante otros factores de desigualdad. El género ha sido la desigualdad históricamente más institucionalizada y ha sido fundamental como referente marco para comparar otras desigualdades (Lombardo, Bustelo, 2010). En los primeros años de despliegue de las políticas de igualdad no había espacio para las mujeres inmigradas puesto que no se contemplaba

\footnotetext{
${ }^{1}$ Para no crear confusión es preciso definir aunque sea de forma breve los términos de transversalidad, discriminación múltiple e interseccionalidad, si bien este último será desarrollado a lo largo del texto. (Informe Genderace, 2010).

El principio de transversalidad se introdujo en el año 1995 en el marco de la Conferencia de Beijing y significó un cambio en la orientación de las políticas al ser recogido más tarde por la Unión Europea. Parte de la consideración que las acciones, las políticas y los programas tienen resultados diferentes para los hombres que para las mujeres. Por este motivo la perspectiva de las mujeres debe ser considerada en el diseño, la implementación, el seguimiento y la evaluación de las políticas y los programas en todos los ámbitos con el objetivo que hombres y mujeres se beneficien por igual y la desigualdad no se perpetúe. Incluye actividades específicas y acciones positivas porque las posiciones de partida para hombres y para mujeres no son iguales.

Discriminación múltiple. Hace referencia a las diferentes situaciones en las que cada persona puede sufrir discriminación. Se puede interpretar como la acumulación en un mismo individuo de diferentes experiencias de discriminación.

Interseccionalidad. Hace referencia a la situación en la cual una clase concreta de discriminación interactúa con dos o más grupos de discriminación creando una situación única. Dentro del contexto académico es el método de análisis sociológico que permite interrogarse sobre la reproducción institucional de la desigualdad.

La transversalidad y la interseccionalidad no son herramientas excluyentes en la intervención para superar la desigualdad de género. Por el contrario se complementan. La interseccionalidad contribuye a diseccionar con más precisión las diferentes realidades en las que se encuentran las mujeres y por lo tanto puede mejorar la acción política.
} 
la diversidad. La categoría "mujer" era el sujeto que orientaba las intervenciones desde todos los ámbitos: trabajo, salud, orientación sexual, etc.

Los instrumentos políticos para desplegar las políticas han sido la legislación y los planes de igualdad que durante años se han considerado los instrumentos exclusivos de articulación de las políticas de igualdad (Bustelo, 2008). La estrategia adoptada para hacer efectiva la igualdad de género ha sido la transversalidad con la combinación de acciones positivas como la manera de contribuir a erradicar la desigualdad histórica de las mujeres y establecer mecanismos institucionales sólidos necesarios para consolidar la igualdad de género en el ámbito político y las administraciones. La perspectiva de género tenía que estar contemplada en los ámbitos de decisión política para poder ser incorporada a todas las áreas de intervención en la construcción de la sociedad

El desarrollo de las políticas de igualdad en España ha sido importante. Desde la creación del Instituto de la Mujer en 1983, de la Secretaría General de las Políticas de Igualdad en 2004, hasta el Ministerio de Igualdad en 2008 de breve existencia, la igualdad de género ha experimentado un impulso considerable. Impulso que se ha hecho latente a través de la creación de estructuras de Igualdad en las principales comunidades autónomas y el diseño específico de planes de igualdad.

Las políticas en Europa mientras tanto, se van adaptando a los cambios demográficos que experimentan los países europeos fruto del asentamiento de población inmigrada y el carácter estructural de esta realidad. Cambios demográficos que configurarán una nueva composición de las sociedades europeas y comportarán cambios económicos y culturales y nuevas necesidades a las cuales habrá que dar respuesta desde las instituciones. La Directiva 2000/43/CE ${ }^{2}$ obligaba a los Estados miembros a elaborar disposiciones para prevenir la discriminación y favorecer un trato igualitario. La Declaración final de la Conferencia Mundial contra el Racismo, la Xenofobia y la Intolerancia firmada en Durban en 2001 recogía que la discriminación, el racismo, la xenofobia y la intolerancia afectan de manera diferente a las mujeres. Desde el año 2000 diferentes directivas europeas antidiscriminación reconocen la discriminación que sufren las mujeres. Mientras, el Pacto Europeo por la Igualdad entre Hombres y Mujeres firmado en $2006^{3}$, integraba en sus objetivos la

${ }^{2}$ El Tratado de Amsterdam aprobado por los 15 países miembros de la Unión Europea en el año 1997 establecía que la igualdad tiene que ver con combatir la discriminación por motivos de sexo, origen racial y étnico, discapacidad, edad, religión y orientación sexual.

${ }^{3}$ Es significativo hacer constar que ese mismo año se creaba el Instituto Europeo de la Igualdad de Género que tiene por objetivo único la igualdad de género y no contempla otras desigualdades. Generó polémica y controversia en el seno de los países miembros la creación de un organismo que se dedicaba en exclusiva a la igualdad de género. Con un presupuesto asignado de 52,5 millones de euros para el período 2007-2013, en la 
doble discriminación que sufren las mujeres inmigrantes o pertenecientes a minorías étnicas.

España donde el impacto de la inmigración se produce más tardíamente que en Europa, debe ir desarrollando políticas para hacer frente a las desigualdades por motivos de origen con el fin de promocionar el diálogo intercultural y la inclusión de las personas inmigradas.

En esta coyuntura el origen étnico, la raza, ha sido la otra desigualdad que ha recibido atención institucional. El género ${ }^{4}$ primero y luego la raza han sido las dos desigualdades más ampliamente tratadas desde la política española. El resto de desigualdades, como en Europa, han tenido un tratamiento más reciente y por tanto se han acometido de forma menos exhaustiva. En el año 2003 se crea el Consejo para la promoción de la Igualdad de Trato y no Discriminación de las personas por motivos de origen racial o étnico adscrito al Ministerio de Trabajo y Asuntos Sociales hasta el año 2008. A partir de ese año pasa a ser competencia del Ministerio de Igualdad a través de la Dirección General contra la Discriminación, de la Secretaría General de Políticas de Igualdad. Esta evolución parece mostrar el camino que han seguido las políticas de igualdad hacia la diversidad (Lombardo, Verloo, 2009) y el paso de un enfoque unitario a otro múltiple que parece ser la tendencia de las políticas de igualdad en la actualidad. Las directrices europeas apuntan hacia la creación de un organismo único anti-discriminación que aparte del sexo y la raza considere otras desigualdades, ante el establecimiento de organismos individuales encargados de cada desigualdad.

Considerar además del género, otras desigualdades exige pasar de un enfoque unitario a un enfoque que ha de integrar desigualdades múltiples que incluyen

actualidad su actividad es baja y su existencia es casi testimonial aunque se mantenga activo de manera oficial. Es relevante que sobre las mujeres inmigradas lo único que se aborda es la realización de un estudio, entre diciembre de 2011 y noviembre de 2012, para conocer cuál es la realidad sobre la mutilación genital femenina en los 27 países miembros más Croacia y establecer estrategias de futuro.

http://www.eige.europa.eu/content/news-article/study-to-map-the-current-situation-and-trends-of-femalegenital-mutilation

Mientras, el nuevo pacto para la Igualdad de género, La Estrategia para la Igualdad entre mujeres y hombres 2010-2015, hace constar que la Comisión Europea debe promover la igualdad entre sexos en todas las iniciativas relacionadas con la inmigración y la integración de los inmigrantes.

${ }^{4}$ Hay que tener en cuenta, no obstante, que la desigualdad de género es estructural y está presente en el resto de desigualdades susceptibles de ser causa de discriminación, entre las cuales la raza y el origen étnico, la discapacidad, la edad, la religión y/o creencias y la orientación sexual. 
primero la raza y la clase social, luego en lugar de la clase social lo harán la edad, la religión o creencia, la discapacidad y la orientación sexual ${ }^{5}$.
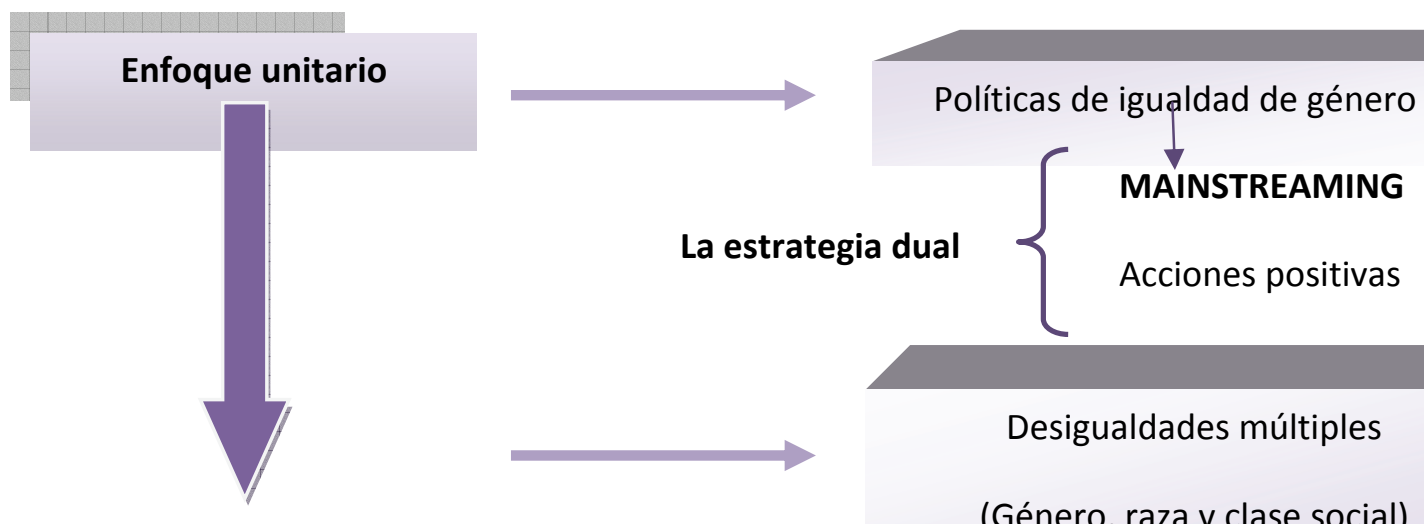

\section{Enfoque integrado}

Desigualdades múltiples

(Género, raza y clase social)

Políticas antidiscriminación

(Género, raza, discapacidad, orientación sexual, religión o creencia y edad)

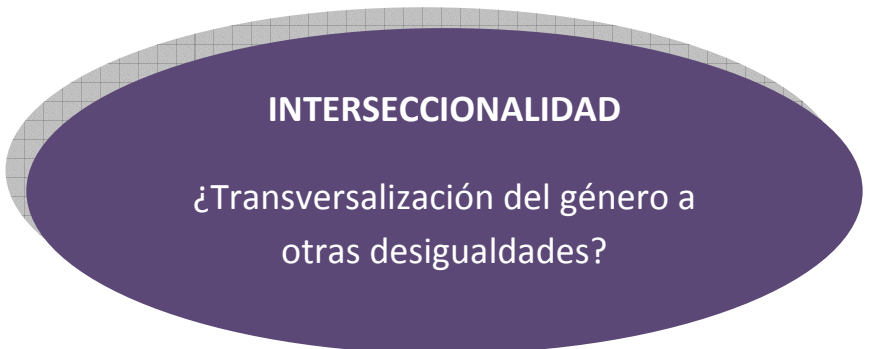

La Comisión Europea adaptó la aproximación múltiple en el año 2007 a raíz del Año Europeo contra la Discriminación. En España todavía no tiene un reconocimiento legislativo, pero los cambios sociales consecuencia de la inmigración, la dependencia de las personas mayores y la discriminación por razón de género han

\footnotetext{
${ }^{5}$ Reconocidas con carácter legal mediante la Ley 51/2003 de 2 de diciembre de igualdad de oportunidades y no discriminación y accesibilidad universal a personas con discapacidad y la ley 13/2005 de 1 de julio de modificación del Código Civil sobre el derecho a contraer matrimonio, respectivamente.
} 
puesto en evidencia que hay desigualdades y discriminąciones múltiples que interseccionan entre sí. Las referencias en el discurso político a otras desigualdades más allá del género, son muy recientes y han sido las políticas de Igualdad las líderes, incorporando en primer lugar la diversidad que va tomando importancia como eje de desigualdad a través de la raza.

La estructura sobre la que se sustenta la consideración de otras desigualdades no obstante, depende de las políticas de igualdad hasta la actualidad. La tendencia, de acuerdo con las orientaciones europeas, parece que es caminar hacia un único marco antidiscriminación como lo atestigua el anteproyecto de Ley Integral para la Igualdad de trato y la no discriminación que fue sometido a un proceso de revisión por parte de las entidades de la sociedad civil y administraciones supralocales relacionadas con la prevención de las discriminaciones y / o desigualdades que afectan a diferentes grupos sociales (ONGs, Áreas de las Diputaciones, Sindicatos, etc.). La Ley perseguía consolidar legislativamente la igualdad y garantizar los derechos existentes y concretamente se dice que vendría a reforzar, acumulando sus efectos a los de la LO 3/2007 sobre la Igualdad efectiva entre mujeres y hombres. La Ley, según se hace constar, amplía y mejora la tutela judicial y la defensa administrativa en materia de igualdad de trato y no discriminación por razón de sexo. Incorpora, además, el enfoque múltiple al reconocer además del sexo, la no discriminación por razón de nacimiento, origen racial o étnico, sexo, religión, convicción u opinión, edad, discapacidad, orientación o identidad sexual, enfermedad, lengua o cualquier otra circunstancia personal o social. La Ley se contemplaba con carácter integral y tenía en cuenta la aplicación transversal de la igualdad de trato en la formulación, ejecución y evaluación de las políticas públicas y las acciones positivas. En la estrategia estatal para la igualdad de trato y no discriminación se señalaba que se prestaría especial atención a las discriminaciones múltiples que por su naturaleza suponen un ataque más grave al derecho a la igualdad. La Ley dependía del actual Ministerio de Sanidad, Política Social e Igualdad.

${ }^{6}$ Bastante avanzado en la consideración de las desigualdades múltiples ha sido el gobierno de la Generalidad de Cataluña y específicamente desde las políticas de igualdad: el V Plan de Políticas de Mujeres del año 2005 definía la identidad de los grupos de mujeres según su experiencia social en torno a la discriminación, la exclusión, o la violencia y resaltaba la necesidad de "visibilizar la diversidad identitaria de las mujeres que contempla el origen, la etnicidad, la edad, la orientación sexual, el modelo de familia, su tiempo de vida, sus prioridades" y en la misma línea hay que interpretar el Plan Interdepartamental para la no discriminación homosexual y las personas transexuales del año 2006. Para algunas autoras en España es necesario diferenciar entre el nivel nacional y regional en relación al tratamiento de las desigualdades desde el ámbito de las políticas y destacan que en las Comunidades Autónomas las organizaciones de la sociedad civil han contribuido a mejorar la respuesta pública ante los cambios (Bustelo, Forest, 2009). 


\section{GÉNERO Y DIVERSIDAD. DISCRIMINACIONES MÚLTIPLES Y APROXIMACIÓN INTERSECCIONAL. UN RECORRIDO DESDE EL CONTEXTO ACADÉMICO}

La igualdad, las acciones positivas y la interseccionalidad fueron tratadas desde las políticas y los movimientos por los derechos civiles en EEUU y Canadá desde un contexto anglosajón (Scrinzi, 2008). En ese contexto las mujeres afroamericanas criticaban al feminismo abanderado por las mujeres blancas, que no respondía a sus necesidades, ni reconocía otras desigualdades aparte del género. El cuestionamiento de un feminismo que sólo daba respuesta a los intereses de unas determinadas mujeres, articulado en la cultura y valores de Occidente, originó que ya desde los años 80 se empezara a gestar un feminismo que incorpora otras realidades desde culturas diferentes a la occidental. Es lo que se conoce como "feminismo periférico" o "feminismo de frontera" (Jabardo, 2008).

El concepto de interseccionalidad lo introduce Kimberlé Crenshaw en la Conferencia Mundial contra el Racismo en Sudáfrica en 2001 . Crenshaw (Crenshaw, 1995), consideró que había categorías como la raza y el género que interseccionaban e influían en la vida de las personas. Para ella el racismo no tenía los mismos efectos sobre los hombres que sobre las mujeres negras y tampoco éstas no vivían las consecuencias del sexismo de igual forma que las mujeres blancas. Partía de una estructura primaria donde interseccionaban a parte de la raza y el género la clase social, a la que se añadían otras desigualdades como la condición de mujeres inmigrantes. Para Crenshaw no se trataba de una suma de desigualdades, sino que cada una de éstas interseccionaban de forma diferente en cada situación personal y grupo social mostrando estructuras de poder existentes en el seno de la sociedad.

Diferenciaba entre la interseccionalidad estructural en relación a las consecuencias de la intersección de varias desigualdades en la vida de las personas y la interseccionalidad política que afecta a la forma en que se contemplan estas desigualdades y cómo se abordan. Así ella denunciaba que las estrategias antirracistas del gobierno americano estaban diseñadas considerando sólo a los hombres negros como categoría general y desconsiderando la perspectiva de las mujeres.

El término de interseccionalidad ha sido formulado desde el feminismo como respuesta a un feminismo occidental exclusivo que no consideraba a las mujeres de otras razas y clases sociales. Su trascendencia entonces, en la interpretación de las

\footnotetext{
${ }^{7}$ Aunque es un término muy reciente sus antecedentes hay que buscarlos en los movimientos de las mujeres negras en los EEUU a finales del siglo XIX y en los movimientos postcoloniales.
} 
condiciones de vida de las mujeres inmigradas, es importante porque incorporó otra dimensión del género desde la perspectiva feminista.

Los primeros estudios e investigaciones sobre las mujeres inmigradas en España, liderados por mujeres que comparten una perspectiva feminista, ya incorporan la consideración de desigualdades múltiples para interpretar sus condiciones de vida, mostrar el contexto en el que se sitúan y orientar las políticas sobre las intervenciones.

Desde el inicio, el género es la primera desigualdad que se contempla y que interactúa con la categoría inmigrada. Se habla de la mujer de origen marroquí señalando la necesidad de considerar a las mujeres con personalidad propia, atendiendo a sus necesidades y aspiraciones específicas y se insta a las políticas de integración a contemplar la igualdad de oportunidades y trato entre hombres y mujeres. (Losada, 1993).

Se piensa la situación de subordinación de las mujeres inmigradas a partir de la opresión racista, por razón de clase social y por diferencia sexual (Solé, 1994) y se entiende su situación desde una dimensión multidimensional que sitúa la clase social, la raza y el género. La interacción entre estos ejes de desigualdad se contempla como una necesidad para aproximarse y entender sus realidades (Colectivo Ioé, 1998).

En la misma línea interpretativa se incorpora a las mujeres inmigradas a la historia general de las mujeres y se reivindica el reconocimiento de la diversidad a partir de valorar la diferencia de clase, de raza, de edad, de formación cultural de pertenencia a un colectivo minoritario (Nash, 1999).

La situación de las mujeres inmigradas se condiciona a la orientación del modelo de integración y al desarrollo de las relaciones de género, vinculando diversidad y género en la mejora o no de sus condiciones de vida. (Aubarell, Ribas, 1999).

Una vez superada una primera fase de carácter sobre todo descriptivo en tanto era necesario conocer quiénes eran las mujeres inmigradas, como vivían, cuáles eran sus realidades personales y familiares y cuál era el contexto en el que se ubicaban, el discurso académico se hace más complejo y pasa a un nivel más teórico de reflexión e interpretación sobre la desigualdad de las mujeres inmigradas en tanto que sujetos de derecho.

Sobre políticas públicas, género e inmigración, se delimita el marco teórico para enfocar el estudio de las mujeres inmigradas a partir de la intersección de las divisiones de género, clase, etnia, edad como factores estructurales responsables de los niveles de inclusión y exclusión. (Maquieira, Gregorio, Gutiérrez, 2000). 
En el año 2003 se introduce de manera general y pública en el mundo académico y político la triple discriminación (Parella, 2003), género, etnia, clase social (mujer, inmigrante y trabajadora) y se la eleva a la categoría desde la que hay que interpretar, entender y enfocar las intervenciones con las mujeres inmigradas. Hay que aclarar que esta nueva dimensión en la interpretación de la realidad de las mujeres a pesar de ser general, tomaba como referente a las mujeres latinas visiblemente activas en el mercado de trabajo y venía en cierto modo a situar en el centro de atención sobre las migraciones femeninas, a las mujeres autónomas en el proceso migratorio, dentro del mercado de trabajo y situadas en espacios laborales de reproducción doméstica en los hogares españoles. Se puede afirmar que creó tendencia y es una de las líneas principales de investigación académica que se ha mantenido hasta la actualidad (Kofman, 2008).

Desde un ámbito más jurídico se reivindica la incorporación de las mujeres inmigradas a la teoría feminista y se hace hincapié en que el género no es el único factor de exclusión. La interseccionalidad se entiende como el instrumento de utilidad para comprender la forma en que funcionan los sistemas cruzados de exclusión que afectan a las mujeres. (Mestre, 2005).

Por otro lado, se sigue indicando la interacción constante en la dinámica de las relaciones de poder de género y la articulación de la experiencia colectiva de las mujeres como minoría de diferencia cultural (Nash, 2005: 104) y se pone el acento en las consecuencias que tiene para las mujeres la interpretación del fenómeno migratorio desde un modelo exclusivamente masculino.

Hasta el momento actual el discurso académico sobre la inmigración femenina en España se ha ido reconstruyendo desde la perspectiva feminista, con una clara voluntad de mostrar de qué manera se está abordando la ciudadanía de las mujeres inmigradas. Se siguen considerando roles, funciones y participación diferenciada entre unos y otras y esto determina su configuración como ciudadanas desde la legislación y demás disposiciones que las afectan. En este nuevo contexto de interpretación, la interseccionalidad ha ido apareciendo con mucha más frecuencia y en estos momentos está prácticamente presente en todos los discursos sobre mujer e inmigración y no sólo como instrumento de análisis metodológico y de interpretación, sino desde su vertiente práctica de análisis de las políticas públicas.

El eje religioso, simbolizado a través del hiyab, es analizado como un factor que intersecciona en la vida de las mujeres musulmanas con otras desigualdades como el origen, la lengua y su estatus legal. Se conoce como islamofobia de género (Ramírez, 2008:127) y contribuye al reforzamiento de los estereotipos orientalistas que considera a las mujeres atrasadas y oprimidas, reforzando sus dificultades de integración. El hecho religioso actúa sobre las mujeres musulmanas como un 
elemento de más desvalorización en relación a las mujeres de otras procedencias y otras confesiones religiosas.

Se critica que la LO 3/ 2007 sobre la Igualdad efectiva entre mujeres y hombres no tiene una perspectiva interseccional en el momento de abordar las desigualdades que afectan específicamente a las mujeres. Valoración que se hace extensiva al resto de programas para potenciar la integración y la cohesión social que desconsideran las especificidades a las que se enfrenta la población inmigrada femenina en su acceso a recursos y oportunidades (Parella, 2009:221). En el lugar opuesto, es decir como ejemplo de buenas prácticas, se considera el Pacto Nacional para la Inmigración del Gobierno de la Generalidad de Cataluña fruto del trabajo de reflexión conjunta, en aquel momento, entre el Instituto Catalán de las Mujeres y la Secretaría para la Inmigración. Se destaca la importancia de la interseccionalidad para visibilizar la desigualdad en la que se encuentran las mujeres inmigrantes a partir de las medidas legislativas previstas para mejorar la igualdad de género y facilitar la gestión del cuidado: son medidas pensadas desde la consideración de la mujer de manera homogénea y por lo tanto sólo responden a las necesidades e intereses de las mujeres autóctonas.

La aproximación interseccional se utiliza, así mismo, ${ }_{8}$ para interpretar los cambios de la última modificación de la LO de Extranjería 4/2000 sobre derechos y libertades de los extranjeros en España y su integración social, la LO 2/2009 de reforma de la LO $4 / 2000$, que continúa sin contribuir a la construcción de una ciudadanía en

\footnotetext{
${ }^{8}$ La primera Ley de Extranjería, la LO 7/1985 sobre derechos y libertades de los extranjeros en España, no reconocía el derecho a la reagrupación familiar y dejaba a las mujeres en una situación de subordinación e indefensión en tanto quedaban supeditadas a la situación jurídica del cónyuge y tenían muchas dificultades para obtener un permiso de trabajo.

La segunda ley de Extranjería, la LO 4/2000 sobre derechos y libertades de los extranjeros en España y su integración social, supuso un avance en la situación de las mujeres ya que reconocía el derecho a la reagrupación familiar y a una autorización de residencia independiente a la del cónyuge si se rompía el vínculo matrimonial.

Esta ley ha tenido desde el año 2000 sucesivas reformas, la LO 8/2000, la LO 11/2003 de 29 de septiembre, la LO 14/2003 de 20 de noviembre, centradas éstas, sobre todo, en el control de flujos y la lucha contra la llegada de personas de manera irregular, y la LO 2/2009. Esta Ley, la última hasta el momento, a través del Real Decreto 557/2011 por el que se aprobaba el Reglamento para su aplicación ha significado una mejora importante en la situación de las mujeres. Ha permitido que las mujeres cuando son reagrupadas, y los hijos e hijas mayores de 16 años, dispongan de autorización para trabajar vinculada directamente a la autorización de residencia y sin necesidad de ningún otro trámite administrativo; y contempla la concesión de residencia temporal y trabajo por circunstancias excepcionales a mujeres extranjeras víctimas de violencia de género (art. 131-134). Con anterioridad en los casos de violencia se otorgaba una autorización de residencia independiente en el momento de emitir una orden de protección, ahora sólo será necesario un informe del Ministerio Fiscal indicando la existencia de delito. También se ha querido ampliar la protección a las mujeres ya que se contempla la concesión de residencia temporal y trabajo a las mujeres víctimas de violencia y en situación de irregularidad administrativa. Estos expedientes tendrán carácter temporal y la residencia, si se confirma el delito, será de cinco años para la mujer y sus hijos e hijas.
} 
igualdad para las mujeres a pesar de los avances. Las medidas consideradas para tratar a las mujeres víctimas de violencia de género siguen gravitando sobre la exigencia de denuncia y la demostración del delito. Se la quiere proteger pero al mismo tiempo sigue siendo prevalente su condición de extranjera. La legislación favorece la construcción de dos categorías de mujeres: las que lideran la lucha por la igualdad y deciden por todas y las víctimas de las violencias variadas (Mestre, 2010:103).

Un ejemplo práctico de aproximación interseccional ha sido el proyecto Genderace, Uso de las leyes de antidiscriminación racial, Género y Ciudadanía en un contexto multicultural, Informe 2010. Fue financiado por la Comisión Europea y en el mismo participó España representada por la Universidad de Barcelona junto a Bélgica, Reino Unido, Alemania, Bulgaria y Suecia.

El Proyecto tenía como eje central el análisis de la dimensión del género de la discriminación racial. Los resultados han mostrado las especificidades de género que tiene el racismo, que se manifiesta de manera diferente en los hombres y en las mujeres. Así por ejemplo, de acuerdo con las funciones y los roles asignado tradicionalmente al género, las mujeres están más discriminadas en su acceso a los servicios públicos, la educación y la vivienda y los hombres en la justicia y el acceso a los servicios privados en su tiempo libre. Las experiencias diferentes que tienen los hombres y las mujeres ante el racismo responden a la división de tareas y a las diferentes representaciones de unos y las otras socialmente configuradas. Otro dato relacionado es que el racismo se muestra en determinadas comunidades: la rumana, la norteafricana o árabe, personas de origen turco y negras, evidenciando que la discriminación está fundada sobre el género, la etnicidad y la condición socioeconómica, sobre todo. En relación a la respuesta y los comportamientos de las personas ante este tipo de situaciones se observó que las mujeres no denunciaban y relacionaban la discriminación de la que eran objeto con la religión y el origen étnico, pero no con el género. Por parte de las instituciones lo más destacable fue la inexistencia de una respuesta global que integrara las diferentes discriminaciones que sufren las mujeres.

La aproximación interseccional es un nivel de mayor complejidad en la identificación de las desigualdades que actúan sobre las mujeres y sobre grupos específicos de mujeres, en tanto permite comprender a través de las diferentes relaciones que se establecen entre las mismas, cuál es el espacio social que ocupan, cuáles son sus posibilidades de reacción y si los recursos de las administraciones potencian la igualdad o por el contrario perpetúan la desigualdad. No se cuestiona su

\footnotetext{
${ }^{9}$ La profesora Olga Jubany fue la responsable de coordinar el proyecto.
} 
valor como instrumento de análisis sociológico, ni su dimensión estructural en tanto las mujeres ocupan posiciones diferenciadas en relación a los hombres y también entre ellas y se encuentran así, con oportunidades y restricciones estructurales en el momento de desarrollar sus capacidades personales y sociales (Lutz, 2010).

\section{4. ¿QUÉ POLÍTICAS? LA IGUALDAD A DEBATE}

El cambio de orientación a nivel político hacia un tratamiento integrado de las desigualdades no está exento de controversia y está generando un debate intenso en el ámbito del feminismo, tanto a nivel filosófico y conceptual, como a nivel operativo desde la formulación de políticas.

Desde el ámbito específico de la igualdad de género no se cuestiona que hay desigualdades estructurales que actúan de manera diferente y con diferente intensidad sobre las personas y grupos sociales, configurando condiciones de vida. Lo que se cuestiona es que a nivel conceptual no es posible tratar de igual manera diferentes desigualdades porque los mecanismos y las estructuras que originan las desigualdades no son similares (Verloo, 2008) . Las actuaciones que en esta dirección se están desarrollando en Europa apuntan a la superposición de desigualdades, sin que se hayan establecido criterios para determinar las relaciones y las dependencias que se establecen entre ellas.

El enfoque múltiple tal y como se está interpretando, es simplista porque parte de la equivalencia de desigualdades y entiende la desigualdad a partir de su efecto en la discriminación. La manera de abordarla entonces, es garantizar la igualdad de trato. No se considera que hay factores estructurales que están en la base de las desigualdades (Bustelo, 2008), y por tanto la desigualdad sólo se trata de forma parcial a través de sus efectos. Considerar la discriminación y favorecer la igualdad de trato no necesariamente implica hacer frente a las desigualdades, ni mucho menos erradicarlas. Las políticas antidiscriminación tienen un carácter reactivo y no proactivo (Bustelo, 2009), plantean la desigualdad como un problema individual en lugar de hacerlo como un problema estructural e institucional.

Si se analiza el caso español hasta el momento actual las desigualdades se han tratado separadamente (género, diversidad, discapacidad, orientación sexual, edad) y no desde la complejidad en la que viven las personas (Bustelo, 2008). En este marco

\footnotetext{
${ }^{10}$ Verloo elabora un cuadro comparativo entre cuatro categorías sociales que se vinculan con desigualdad: género, raza o etnia, orientación sexual y clase y muestra las diferencias entre ellas en relación al contexto en el cual actúan, cómo se han construido socialmente, que tratamiento político reciben, etc. y la conexión existente entre ellas. Esto debería de orientar las estrategias políticas para tratarlas.
} 
la igualdad de género ha sido prioritaria, pero con el cambio de enfoque el género puede perder "categoría", existe el riesgo de que se diluya entre otras desigualdades sociales. Por otro lado disponer de organismos integrados puede originar menos recursos y mecanismos más frágiles para abarcar la complejidad de desigualdades múltiples (Lombardo, Verloo, 2009).

A nivel operativo el debate se centra en determinar cuál debe ser la metodología o metodologías más adecuadas para tratar las desigualdades / discriminaciones múltiples. Parece haber acuerdo, a nivel académico, que la mejor manera de tratar las desigualdades múltiples entre las que se encuentra el género y la raza o la etnia, es la teoría feminista de la interseccionalidad. Hay sin embargo, apreciaciones diferentes:

Dentro del estudio de las migraciones femeninas se considera la perspectiva interseccional como el método más sofisticado en la identificación de manifestaciones concretas de la desigualdad, en tanto desde otros patrones analíticos que sólo tuvieran en cuenta las diferencias de género no sería posible identificar (Parella, 2010).

Dentro del estudio de las políticas de igualdad de género, la transversalización de la diversidad puede ser una estrategia adecuada para tratar las desigualdades múltiples. Sin ser una simple adaptación de la transversalidad de género puede orientar y servir de experiencia. Se defiende como estrategia porque permitiría tratar las múltiples ubicaciones, intersecciones y mecanismos de las desigualdades múltiples, tratar las desigualdades a escala de las estructuras y las instituciones y también tratar el sexismo, el racismo, la explotación de clase, la homofobia, la discriminación por razón de edad, etc., en los procesos de elaboración de las políticas y en las propias políticas.(Verloo, 2008: 24).

La crítica está dirigida a la formulación de políticas. En Europa parece que la interseccionalidad no se está aplicando porque no se está considerando la intersección entre las desigualdades, ni tampoco hay evidencia de que se esté aplicando la transversalidad de género en otras desigualdades. El Instituto para la Igualdad de Género no se refiere en sus objetivos a las intersecciones entre el género y el resto de desigualdades, ni ha sido un tema a tratar. En general la forma como se está tratando la discriminación múltiple en Europa no refleja que se quiera hacer desde la

\footnotetext{
${ }^{11}$ Es ilustrativa al respecto la convocatoria de subvención realizada por la Comisión Europea en este año, dentro del programa Progress, 2007-2013 en su sección 4 sobre antidiscriminación y diversidad. Se trata de dar soporte a las actividades nacionales que tengan por objetivo combatir la discriminación y favorecer la igualdad. Las actividades deben estar orientadas por lo menos a uno de los cinco motivos de discriminación: raza y origen étnico, discapacidad, edad, religión o creencia y orientación sexual. La perspectiva de género deberá ser contemplada con carácter general. Se abordan las distintas discriminaciones a título particular básicamente y aunque la dimensión de género parece que corta transversalmente al resto de motivos de discriminación, la aproximación interseccional no se tiene en cuenta cómo método de intervención social.
} 
intersección de desigualdades, que por otra parte exige estructuras de coordinación (Lombardo, Verloo, 2009).

En España la discriminación múltiple y la interseccionalidad son todavía de emergencia débil y se sitúan en el debate entre el género y la raza principalmente, al menos hasta el momento de redacción del Anteproyecto de Ley Integral para la igualdad de trato y no discriminación. Como ejemplo el Anteproyecto reconoce las discriminaciones múltiples y el enfoque integral, pero no menciona en ningún momento la interseccionalidad. Considera que la discriminación múltiple tiene lugar cuando concurren o interactúan diversas causas de las previstas en la Ley, generando una forma específica de discriminación. No acaba de quedar clara cuál es su filosofía de fondo, pero el texto de la Ley parece indicar que tendrá más peso la consideración de la discriminación que la desigualdad y por tanto la interseccionalidad tal y como la define la teoría feminista, no es contemplada. Por otra parte la desaparición del Ministerio de Igualdad corrobora la tendencia de articular en España un único órgano de atención a las diferentes desigualdades, ante la existencia de organismos específicos. Para algunas expertas en políticas de igualdad esto es un claro ejemplo de que el contexto político actual no es favorable a la igualdad de género (Lombardo, Verloo, 2009).

\section{CONCLUSIONES}

Si las migraciones han sido un fenómeno estructural en las sociedades europeas, la promoción de las políticas de género ha contribuido a configurar una nueva realidad social. La igualdad de género reconocida como un derecho fundamental, ha hecho emerger desigualdades poco consideradas y ha originado que se deriven recursos y medios materiales y humanos para superarlas, ha cambiado la manera de enfocar las intervenciones dirigidas a la población y ha configurado un nuevo modelo donde las mujeres y los hombres ya no se definen por su sexo, sino como seres humanos con las mismas posibilidades y oportunidades de intervenir en la vida pública. En este intersticio se sitúan las mujeres inmigradas. La emergencia de las políticas de igualdad en Europa y en España y legislaciones que han elevado la igualdad entre mujeres y hombres a un derecho fundamental han significado recursos específicos para la promoción y la mejora de las capacitaciones de las mujeres. Ha sido el momento en el que la inmigración femenina ha empezado a ser considerada en función de su sexo.

El paso hacia un enfoque múltiple de las desigualdades desde la perspectiva feminista, donde el género se sitúa en el centro como eje principal, ha sido un paso adelante en el análisis de las realidades de las mujeres inmigradas y la interseccionalidad como método de análisis permite sobre todo medir el impacto de las políticas y su efectividad sobre la superación o el reforzamiento de las 
desigualdades. Para el feminismo ha sido un cambio positivo que no debe segregar, sino sumar esfuerzos en la defensa de derechos. Es la manera lógica de integrar la diversidad dentro del feminismo y una evolución consecuente en el esfuerzo de adaptación a las dinámicas económicas y sociales de los países. Dinámicas que configuran sectores sociales nuevos en la escena pública, nuevas problemáticas, necesidades y retos pero también nuevas oportunidades.

La interseccionalidad ha originado un debate muy interesante desde el feminismo y la reflexión académica, que se sitúa entre la universalidad del género como eje que debe regular las políticas de igualdad, y la consideración de que la desigualdad de género tiene diferentes acepciones que hay que integrar para dar respuesta a la heterogeneidad de las situaciones que viven las mujeres. La inmigración ha contribuido a abrir un debate que tiene sus antecedentes en los años 60 dentro de los movimientos feministas de las mujeres negras en EEUU. La interseccionalidad aparece como una herramienta útil para conocer con más exactitud el nivel de intensidad con respecto a la desigualdad que afecta a las mujeres en función de una serie de variables y actuar en consecuencia derivando más o menos recursos sobre determinados grupos sociales. Desde esta definición es un instrumento eficaz que permite actuar más desde la equidad, que desde la igualdad propiamente entendida. De manera general las acciones para la igualdad es evidente que no tienen el mismo impacto según las diferentes situaciones que viven las personas. La dificultad en torno a la interseccionalidad se encuentra justamente en la necesidad de fragmentar la realidad. Si el carácter universal del género puede obviar y dejar al margen grupos sociales por tener realidades más complejas, la interseccionalidad es un método costoso y difícil en su aplicación. Dentro del marco del trabajo de las administraciones públicas resulta prácticamente inviable su implementación, tal y como están configuradas. Realizar aproximaciones interseccionales sobre un eje parcial de desigualdad, por ejemplo la manifestación del racismo sobre hombres y mujeres, no contribuirá a mejorar sustancialmente la situación de determinados grupos de mujeres, como es el caso de las mujeres inmigradas. Contrariamente puede reproducir desigualdades por el hecho de focalizar y resaltar el hecho cultural diferencial. La interseccionalidad para su eficacia en el diagnóstico que se convierte en la parte más importante del proceso, debe valorar y analizar todas las situaciones particulares (no sólo ser mujer inmigrada, también edad, nivel de formación, etc.), que puedan ser significativas de producir desigualdad social, sin perder de vista el carácter estructural de la desigualdad de género.

Las líneas políticas europeas y española parecen reconocer el enfoque múltiple pero el género deja su centralidad para compartirla con otras desigualdades como la raza o la etnia, la edad, la religión, la orientación sexual y la discapacidad. La interseccionalidad además, no está considerada en la producción de políticas, ni a nivel europeo, ni a nivel estatal, aunque en algunos planes y programas se utilice el término de forma incorrecta en cuanto a su significado. 
Las críticas van dirigidas a que las discriminaciones son consideradas de manera idéntica, actúan con la misma intensidad y por tanto se originan en base a unos mismos mecanismos y procesos. La discriminación es la manifestación de la desigualdad y entonces se actúa sobre el efecto pero no sobre la causa.

El alcance de esta nueva interpretación de las desigualdades va a mi juicio más allá del futuro de las políticas de igualdad de género. Significa un cambio radical en la forma de enfocar las diferencias sociales fruto de procesos económicos y sociales determinados. Con la nueva interpretación no sólo desaparece la dimensión estructural de la desigualdad de género, sino que promueve la desaparición de la conciencia de género. Las nuevas políticas antidiscriminatorias obvian el concepto de desigualdad absolutamente válido para reflejar situaciones comparativas con respecto al uso y acceso a los bienes, participación, trabajo y condiciones de vida, entre grupos sociales y entre mujeres y hombres. En tanto que prioriza el individuo por encima de las estructuras tiende a la neutralización de la acción colectiva y la reacción social. Es un enfoque disgregador de lo colectivo. En relación a los efectos sobre las mujeres inmigradas como señala Wassyla Tamzali las políticas europeas están pasando de las formas universales de crítica social a un enfoque culturalista y diferencial con las consecuencias que conlleva sobre la manera de actuar ante las causas de la desigualdad y sobre las posibilidades de cambio social.

Las políticas de igualdad de género no han tenido tiempo suficiente para desplegarse en su totalidad. La transversalidad como la estrategia para superar la desigualdad que sufren las mujeres por el hecho de serlo, no ha llegado a ser una estrategia consolidada e incorporada a todas las administraciones, ni desde todos los ámbitos. Sin embargo, los avances y las transformaciones han sido muy importantes, sobre todo porque han dignificado la vida de las mujeres no sólo desde las diferentes normativas y medidas aprobadas, sino por el hecho de otorgar voz a las mujeres en la propia definición. Si bien existen limitaciones importantes desde las propias políticas con respecto a la interpretación de las relaciones de género y de la igualdad y también es verdad que hay una necesidad estratégica de incorporación de las mujeres al mercado de trabajo asalariado, es incuestionable la presencia de las mujeres en espacios donde hasta hace poco estaban excluidas, así como la superación de muchas barreras que afectaban a su vida laboral, profesional y personal y la existencia de recursos y servicios a disposición de las mujeres antes inexistentes. Las mujeres inmigradas, en tanto que mujeres, se han visto igualmente beneficiadas, encontrando las que ahora llegan a España una situación muy diferente a la que encontraban las mujeres 20 años atrás. Si se hace efectivo el cambio de orientación perderán calidad en la atención a sus necesidades como mujeres. Si son consideradas por su origen favorecerá que continúen asociadas a la diferencia cultural, perpetuando la visión etnicista y excluyente. La etnia o la raza no es ninguna condición universal de desigualdad. La desigualdad aparece cuando se dan otras condiciones como la precariedad económica y las pocas opciones de promoción social y personal. Las 
mujeres inmigradas sufren las desigualdades inherentes a un sistema patriarcal de organización social y con igual intensidad las que se derivan de su condición social. Priorizar que es discriminada en función de su origen, etnia o raza, esconde otras desigualdades estructurales susceptibles de poder ser modificadas y sigue dejando a las mujeres encerradas entre límites culturales.

La desigualdad por razón de género no es equiparable a otras desigualdades, las desigualdades no son similares entre sí, y no puede tener el mismo tratamiento. En cuanto a las mujeres inmigradas no se puede obviar que siguen ocupando los puestos de trabajo con peores condiciones económicas, pocos derechos laborales y tienen muchas dificultades todavía para su promoción social y personal, como por el ejercicio de derechos. Las mujeres constituyen un colectivo con una situación de desventaja histórica, las mujeres migradas constituyen un colectivo dentro de un colectivo y son la alteridad dentro de la alteridad.

Se desconoce el peso que tendrá el género y la raza en el nuevo enfoque de las discriminaciones múltiples como desigualdades principales inicialmente, en su articulación. Esto es lo que determinará la orientación de las políticas y permitirá deducir el futuro para las mujeres con mayor o menor optimismo en una coyuntura de crisis e incertidumbre económica.

\section{BIBLIOGRAFIA}

AUBARELL, Gemma, RIBAS, Natàlia. (1999): Quines respostes? Polítiques en clau de gènere en M-A. Roque (dir.) Dona i migració a la Mediterrània occidental. p. 273-295. Barcelona: Institut Català de la Mediterrània

BUSTELO, María (2008): A better performer in gender than in intersectionality. Fourt Pan-European conference on EU politics, http://www.jhubc.it/ecprriga/virtualpaperroom/115.pdf, Riga (Latvia), 25- 27 Sept., 2008

COLECTIVO IOÉ (1998) Mujeres Migrantes en España. Proyectos migratorios y trayectorias de género.

http://www.colectivoioe.org/index.php/publicaciones articulos/show/id/37

CRENSHAW, Kimberlé (1995): Mapping the Margins: Interseccionality, Identity Politics and violence Againts Women of Color en K. Crenshaw; N. Cotanda; C. Peller; K. Thomas (eds.) Critical Race Theory. The key writings that formed the movement. p. 357-383. New York: The New Press

FOREST, Maxime; PLATERO, Raquel (2008) Report Analysing Intersetionality in Gender equality Policies for Spain and the EU. Quing, Quality in Gender+ Equality Policies, Institut for Human Sciences, http://www.quing.eu/files/results/ir_spain.pdf Viena, 2008

JABARDO, Mercedes (2008): Desde el feminismo negro, una mirada al género y la inmigración en L. Suárez, E. Martin, A. Hernández (coords.) Feminismos en la 
antropología. Nuevas propuestas críticas. Congreso de Antropología. p. 39-54 Donostia: Ankulagi Antropologia Elkatea.

http://www.euskomedia.org/PDFAnlt/antropologia/11/06/06039054.pdf

KÓCZÉ,Angéla (2011) La stérilisation forcé des femmes roms dans l'Europe d'aujourd'hui. Genre, modernité et "colonialité" du pouvoir. Cahiers du Gendre, 50, p. 133-152

KOFMAN, Eléonore (2008) Genre, migrations, reproduction sociale et Welfare state.

Un état des discussions. Les cahiers du CEDREF, 16 p.101-124

http://cedref.revues.org/579

LOMBARDO, Emanuela; BUSTELO, María (2010) The political treatment of inequalities in Southern Europe a comparative analysis of Italy, Portugal and Spain. II Congreso Anual Red Española de Política Social, 30 de septiembre y 1 de octubre, Madrid, 2010

Centro de Ciencias Humanas y Sociales, CSIC, http://www.espanetspain.net/congreso2010/paneles/pane15

LOMBARDO, Emanuela; VERLOO, Mieke.: La institucionalización de la interseccionalidad de género con otras desigualdades en la Unión Europea: desarrollos políticos y contestaciones. IX Congreso Español de ciencia política y de

administración,http://www.ucm.es/info/target/Art\%20Chs\%20ES/LombardoVerlo o AECPA09ES.pdf, Málaga, 23-25 septiembre, 2009

LOSADA, Teresa (2003): La mujer inmigrante marroquí en España. Ponencia Congreso Mujeres, democracia y desarrollo en el Magreb. Fundación Pablo Iglesias.

LUZT, Helma (2010): Los límites de la europeidad. Las mujeres inmigrantes en la fortaleza europa en P. Bastida, C. Rodríguez (eds.) Nación, diversidad y género. Perspectivas crítica, p.89-132, Barcelona: Anthropos

MAQUIEIRA D'ANGELO, Victoria.; GREGORIO GIL, Carmen.; GUTIÉRREZ LIMA, Elena (2000) Politicas públicas, género e inmigración en P. Pérez Cantó (ed.) También somos ciudadanas. p. 371-438, Madrid: instituto Universitario de estudios de la mujer. 438

NASH, Mary (1999) Construcció social de la dona estrangera, en M-A. Roque (dir.) Dona i migració a la Mediterrània occidental, p. 235-247, Barcelona: Institut Català de la Mediterrània

- (2005) Inmigrantes en nuestro espejo. Barcelona: Icaria

MESTRE, Ruth (2005): Feminisme, dret i immigració: una crítica feminista al dret d'estrangeria. Valencia: Servei de publicacions Universitat de Valencia (tesi de doctorat)

Universitat de Valencia

http://www.tdx.cat/handle/10803/9855;jsessionid=BCAB26B35E497260D66A8634D F6D50F8.tdx1

- (2010) Mujeres, nueva ciudadanía y trabajo: ¿de qué mujeres hablamos? en M. Freixanet Mateo (coord.) Dones migrades i treballadores. Anàlisi i experiències 
locals contra la desigualtat, p. 75-106, Barcelona: Institut de Ciències Polítiques i Socials

PARELLA Sonia (2003): Mujer, inmigrante y trabajadora: la triple discriminación, Barcelona, Anthropos

- (2009) La gestió política del gènere i la dona immigrant en R. Zapata-Barrero, Políticas y gobernabilidad de la inmigración en España. p. 207-224, Barcelona: Ariel

- (2010): Dones migrades a Catalunya: eines conceptuals per tal d'interpretar els seus patrons de segregació laboral i les seves trajectòries en M. Freixanet Mateo (coord.) Dones migrades treballadores. Anàlisi i experiències locals contra la desigualtat, p. 15-46, Barcelona: Institut de Ciències Polítiques i Socials.

SANNA, Maria Eleonora; VARIKAS, Eleni (2011) Genre, modernité et 'colonialité' du pouvoir, Cahiers du Genre, ${ }^{\circ} 50$, p. 5-15

SOLÉ, Carlota (1994) La mujer inmigrante. Madrid: Ministerio de Asuntos Sociales, Instituto de la Mujer.

SCRINZI, Framcesca (2008) Quelques notions pour penser l'articulation des rapports sociaux de "race ", de classe et de sexe, Les cahiers du CEDREF.p 8199

http://cedref.revues.org $/ 578$

VERLOO, Mieke (2008) Una atenta mirada a les desigualtats múltiples, a les seves interseccions i com es tracten a Europa en E. Bodelón, P. Giménez: Construint els drets de les dones. Dels conceptes a les polítiques local, p.17-36, Barcelona: Diputació de Barcelona 